\title{
Short term coseasonal sublingual immunotherapy reduced the development of asthma in children with hay fever
}

Novembre E, Galli E, Landi F, et al. Coseasonal sublingual immunotherapy reduces the development of asthma in children with allergic rhinoconjunctivitis. J Allergy Clin Immunol 2004;1 14:851-7.

In children with allergic rhinoconjunctivitis (hay fever), does short term coseasonal sublingual immunotherapy reduce the development of asthma and seasonal hay fever symptoms?

\section{METHODS}

$+$

Design: randomised controlled trial

Allocation: $\{$ unconcealed $\}$ *

$Y$

Blinding: blinded \{patients, healthcare providers, and data collectors $\}^{*}$.

I

Follow up period: 3 years.

Setting: 6 paediatric allergy centres in North Central Italy (Emilia, Tuscany, and Lazio).

Patients: 113 children 4-16 years of age (mean age 8 y) with hay fever caused by grass pollen and clinical monosensitisation to grass pollen. Exclusion criteria: asthma, clinical sensitisation to other inhalant allergens, and previous immunotherapy for grass pollen allergy.

$\mathbf{R}_{\mathbf{x}}$

Intervention: sublingual immunotherapy with an extract of mixed grass pollens given in a short term coseasonal protocol $(4 \mathrm{mo}$ each y during pollen season) plus standard symptomatic treatment (active treatment group, $n=54$ ) or standard symptomatic treatment only (control group, $n=59$ ).

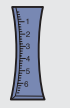

Outcomes: development or recurrence of asthma and hay fever symptoms: for the nose (itching, runniness, blockage, and sneezing), eyes (itching, lacrimation, and redness), and asthma ( $\geqslant 3$ episodes of wheezing breathing difficulty, cough, or both, $\geqslant 1$ wk apart).

$\square$ Patient follow up: 97 (86\%) children (70\% boys).

*Information provided by author.

\section{MAIN RESULTS}

At 3 years, the rate of development of asthma was lower in children in the active treatment group than in the control group (table). Differences in other outcomes were found only after the second year (ie, children in the active treatment group had lower subjective evaluation scores for symptoms [ $p=0.03]$, drugs $[p=0.009]$, and symptoms plus drugs $[\mathrm{p}=0.001])$.

\section{CONCLUSION}

In children with hay fever, short term coseasonal sublingual immunotherapy reduced the development of seasonal asthma and improved hay fever symptoms. Milano, Italy. burastero.samuele@hsr.it

Source of funding: no external funding.
Short term coseasonal sublingual immunotherapy and standard symptomatic treatment (active) $v$ standard symptomatic treatment alone (control) in children with hay fever*

\begin{tabular}{lllll}
\hline $\begin{array}{l}\text { Outcome at } \\
\mathbf{3} \text { years }\end{array}$ & Active & Control & RRR (95\% CI) & NNT (CI) \\
\hline $\begin{array}{l}\text { Development of } \\
\text { asthma }\end{array}$ & $17 \%$ & $37 \%$ & $55 \%(9$ to 78$)$ & $5(3$ to 42$)$ \\
\hline
\end{tabular}

*Abbreviations defined in glossary; RRR, NNT, and $\mathrm{Cl}$ calculated from data in article.

\section{Commentary}

A sthma causes morbidity and mortality in both paediatric and adult populations. ' Prevention of bouts of this disease will result in better quality of life for many individuals and cost savings for the healthcare system. Sublingual immunotherapy is used to treat symptoms of allergic rhinitis, ${ }^{2}$ and may be used to reduce the need for antihistamines and inhalers.

The study by Novembre et al builds on a growing body of evidence that shows that sublingual immunotherapy is safe and effective, ${ }^{3}$ reduces the symptoms of allergic rhinitis, and helps to reduce the development of asthma. The study corroborates the results of previous studies, with 1 key difference: sublingual immunotherapy was coseasonal and not continvous. Intermittent use of sublingual immunotherapy may provide greater acceptance of the treatment and increased cost effectiveness.

The authors point out some weaknesses in the study, such as the lack of blinding of parents. Parental concerns can alter the ability to do a study that may pose an element of risk or hardship on a child. The authors do not provide a description of the training given to healthcare providers, children, or parents about describing symptoms in the diaries and aggressiveness of treatment for symptom relief. This raises concerns about the consistency of reporting of symptoms and supplementary treatment (eg, salbutamol and antihistamines).

In summary, the study by Novembre et al provides further useful evidence that sublingual immunotherapy has a role in the prevention of asthma in children with hay fever. These results will be of interest to healthcare providers in primary health care and specialists in allergy treatment centres or respiratory care.

Roberta Heale, RN (EC), BScN Laurentian University Sudbury, Ontario, Canada

1 Eid NS. Update on National Asthma Education and Prevention Program pediatric asthma treatment recommendations. Clin Pediatr (Phila) 2004;43:793-802.

2 Bowen T, Greenbaum J, Charbonneau Y, et al. Canadian trial of sublingual swallow immunotherapy for ragweed rhinoconjunctivitis. Ann Allergy Asthma Immunol 2004;93:425-30.

3 Passalacqua G, Guerra L, Pasquali $M$, et al. Efficacy and safety of sublingual immunotherapy. Ann Allergy Asthma Immunol 2004;93:3-12. 